\title{
Aplicaciones del láser de baja potencia en Odontología
}

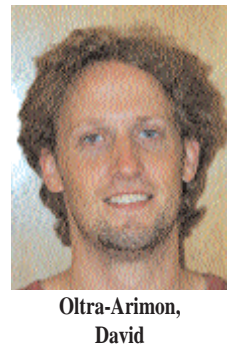

\section{Applications of low level laser therapy in dentistry}

\section{Oltra-Arimon, David* \\ España-Tost, Antonio Jesús** Berini-Aytés, Leonardo*** Gay-Escoda, Cosme ${ }^{\star \star * *}$}

*Odontólogo. Residente del Máster de Cirugía e Implantología Bucal. Facultad de Odontología de la Universidad de Barcelona.

**Médico Estomatólogo. Profesor Asociado de Cirugía Bucal y Profesor del Máster de Cirugía Bucal e Implantología Bucofacial. Facultad de Odontología de la Universidad de Barcelona.

***Médico Estomatólogo. Cirujano Maxilofacial. Profesor Titular de Patología Quirúrgica Bucal y Maxilofacial. Profesor del Máster de Cirugía Bucal e Implantología Bucofacial. Facultad de Odontología de la Universidad de Barcelona.

****Médico Estomatólogo. Cirujano Maxilofacial. Catedrático de Patología Quirúrgica Bucal y Maxilofacial. Director del Máster de Cirugía Bucal e Implantología Bucofacial. Facultad de Odontología de la Universidad de Barcelona. Cirujano Maxilofacial del Centro Médico Teknon. Barcelona.

\section{Correspondencia}

Cosme Gay Escoda

Centro Médico Teknon

Cl. Vilana 12

08022 - Barcelona

E-mail: cgay@ub.edu

http://www.gayescoda.com
Resumen: La utilización de la tecnología láser en Odontología ha tenido una constante evolución y desarrollo. Con este trabajo se pretende hacer una actualización desde un punto de vista crítico, científico y objetivo de los avances y aplicaciones que se han publicado sobre el láser de baja potencia en el ámbito de cada una de las especialidades odontológicas. Asimismo, se exponen sus características, las normas de seguridad necesarias para su utilización, sus efectos secundarios y sus contraindicaciones.

Palabras clave: Láser de baja potencia, Láser terapéutico, Odontología.

Abstract: The use of laser devices in dentistry has undergone a constant evolution and development. The aim of this work is the critical review, from a scientific and objective point of view, of the latest advances and applications reported in the literature regarding low level laser therapy applied to the different dental specialties. Besides, their main features, security advice on its use, adverse effects and contraindications are exposed.

Key words: Low level laser therapy, Therapeutic laser, Dentistry.

\begin{tabular}{ccc}
\hline Fecha recepción & Fecha última revisión & Fecha aceptación \\
$25-4-2000$ & $26-2-2001$ & $19-3-2001$ \\
\hline
\end{tabular}

BIBLID [1138-123X (2004)9:5; septiembre-octubre 477-612]

Oltra-Arimon D, España-Tost AJ, Berini-Aytés L, Gay-Escoda C. Aplicaciones del láser de baja pótencia en Odontología. RCOE 2004;9(5):517-524. 


\section{Introducción}

La utilización del láser en Odontología ha tenido una constante evolución y desarrollo; cada vez son más las especialidades odontológicas en las que se aplican las diferentes variedades de láser ya sea en procesos diagnósticos o terapéuticos. En este aspecto, cada vez hay más profesionales atraídos por esta tecnología y este hecho tiene mucho que ver con los esfuerzos de los científicos para facilitar y optimizar sus amplias utilidades en la clínica dental.

De este modo, se deben distinguir dos grandes grupos de láseres: los de alta potencia o quirúrgicos y los de baja potencia o también denominados terapéuticos (low level laser therapy o LLLT). Los primeros tienen un efecto térmico ya que son capaces de concentrar una gran cantidad de energía en un espacio muy reducido y ello se demuestra por su capacidad de corte, coagulación y vaporización. Por otro lado, los láseres de baja energía carecen de este efecto térmico ya que la potencia que utilizan es menor y la superficie de actuación mayor, y de este modo el calor se dispersa; sin embargo producen un efecto bioestimulante celular. Su aplicación fundamental es para acelerar la regeneración tisular y la cicatrización de las heridas disminuyendo la inflamación y el dolor. Los más conocidos son el de Arseniuro de Galio (Ga,As, láser pulsado con longitud de onda de 904 nm), el de Arseniuro de Galio y Aluminio transmisible por fibra óptica (Ga,Al,As con longitud de onda de $830 \mathrm{~nm}$ ) y el de Helio-Neón (HeNe con longitud de onda de 632,8 nm), este último dentro del espectro visible, concretamente el rojo.

El láser blando es aquel láser de

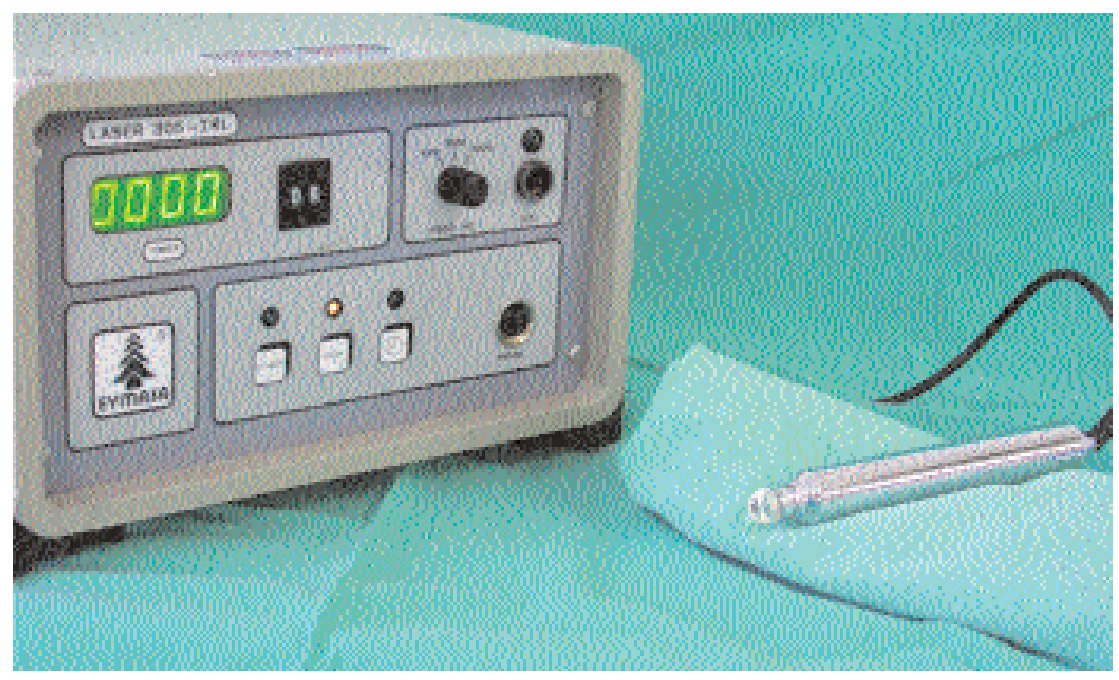

Figura 1: Láser de Ga,Al,As y pieza de mano.

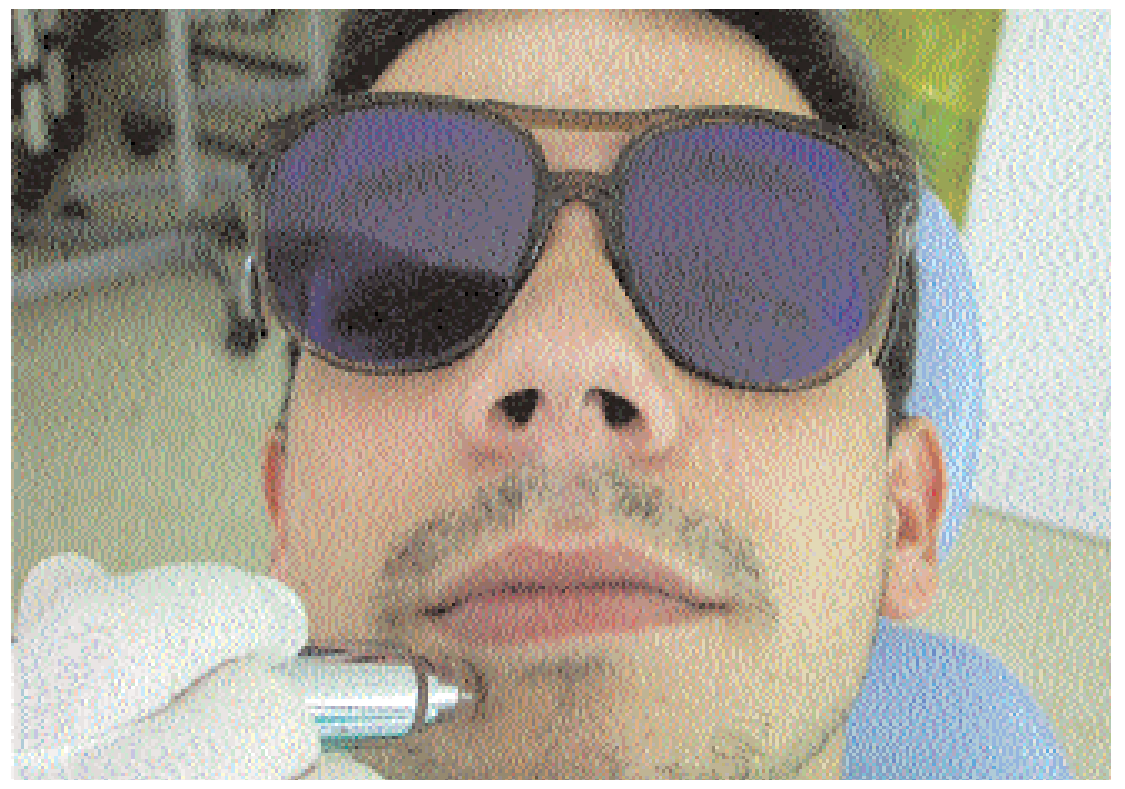

Figura 2: Aplicación del láser de Ga,Al,As en el dermatomo del nervio dentario inferior tras demostrarse la aparición de parestesia en el labio inferior.

baja energía que emite en la región del espectro rojo o del infrarrojo cercano, con una potencia media desde $50 \mathrm{~mW}$ hasta $1 \mathrm{~W}^{1}$ y que no produce efecto térmico. Así pues, al trabajar con una potencia baja, no estará indicado en el ámbito quirúrgico.

Haciendo un viaje por la historia del láser blando debemos situar su origen en 1917, época en la que Einstein describió el concepto teórico de la emisión estimulada de la radiación². Enumerándolos por orden cronológico, el láser de He-Ne fue introducido en 1960 y dos años más tarde aparecieron los láseres de semiconductores. Fue en 1966 
cuando Mester, cirujano y radiólogo, pionero en la investigación de esta tecnología de baja radiación, aplicó el láser de baja potencia para acelerar el proceso de cicatrización dentro del tratamiento de las úlceras crónicas.

Todavía hoy en día no se conoce perfectamente su mecanismo de acción pero se cree que modula el comportamiento celular sin incrementar significativamente la temperatura tisular. De este modo, su actividad sobre los tejidos no obedece a efectos térmicos, sino a la interacción de las ondas electromagnéticas de esta radiación con las células. La energía es absorbida donde la concentración de fluidos es mayor; por lo tanto habrá una mayor absorción en los tejidos inflamados y edematosos, estimulando las numerosas reacciones biológicas relacionadas con el proceso de reparación de las heridas.

Se produce una interacción entre las células y los fotones irradiados (reacción fotoquímica); la célula absorbe la energía del fotón y ésta es transferida a las distintas biomoléculas, que a su vez estimulan otras biomoléculas. La energía transferida, que dependerá del poder de penetración del haz de energía, provoca un au-mento de la energía cinética activando o desactivando enzimas u otras propiedades físicas o químicas de otras macromoléculas principales. Los mecanismos exactos que fundamentan este proceso aún son desconocidos y actualmente son motivo de estudio por parte de la comunidad científica; por este motivo, para algunos autores su uso es muy empírico y fruto de estudios observacionales.

En el Máster de Cirugía Bucal e Implantología Bucofacial de la Facultad de Odontología de la Universidad de Barcelona disponemos de una unidad de

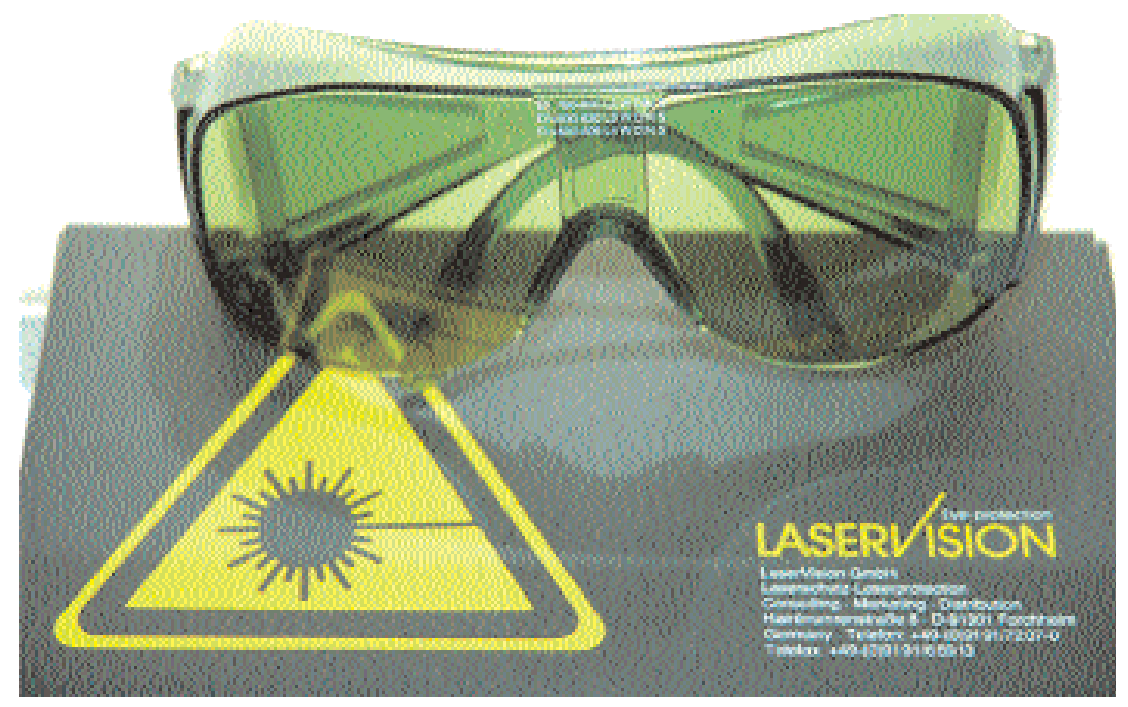

Figura 3: Gafas de protección para cuando se emplea el láser.

láser de Arseniuro de Galio y Aluminio (Ga,Al,As) con el que aplicamos tratamientos de bioestimulación a los pacientes que lo requieran como es el caso, entre otros, de la regeneración nerviosa tras la lesión de los nervios dentario inferior y lingual al demostrarse la recuperación de la sensibilidad del territorio que inervan (figs. 1 y 2).

\section{Clasificación}

El láser de baja potencia está considerado como un láser que puede incluirse desde la clase II hasta la clase Illa según la potencia utilizada y los criterios de seguridad requeridos por el Instituto Americano Nacional de Estandarización (ANSI) ${ }^{3}$. Estos equipos pueden producir daños en el globo ocular si el haz de luz incide directamente en él, por lo que tanto el paciente como el operador o cualquier persona dentro del área de aplicación, deberán ir perfectamente protegidos ya que el haz de energía láser puede ser reflejado por instrumentos próximos a la zona operatoria o incluso por los propios tejidos.

El principal riesgo que supone la manipulación de radiaciones visibles o infrarrojas con longitudes de onda entre los 400 y los $1400 \mathrm{~nm}$ es la posibilidad de dañar la retina. Esto provocaría una reducción del campo de visión y, si la exposición es elevada, la lesión podría ser irreversible ${ }^{4 * *}$.

Por este motivo es imprescindible el uso de gafas protectoras especiales (fig. 3), tanto para el paciente como para el profesional, sus ayudantes y el personal auxiliar, evitando las superficies reflectantes (instrumentos metálicos, espejos o incluso obturaciones dentarias metálicas pulidas) cerca de la zona operatoria. También debe señalizarse adecuadamente (carteles azules con letras blancas) las áreas donde se trabaja con láser.

Existen varias unidades comercializadas de láseres de baja energía de entre las cuales destacaremos el láser de Arseniuro de Galio y Aluminio ( $\mathrm{Ga}, \mathrm{Al}, \mathrm{As}$ ), el láser de Arseniuro de Galio $(\mathrm{Ga}, \mathrm{As})$ y el 
láser de Helio-Neón.

- El láser de GaAlAs es un láser contínuo con una longitud de onda de $830 \mathrm{~nm}$, que puede trabajar con una potencia máxima de $10 \mathrm{~W}$ y es transmisible por fibra óptica.

- El láser GaAs es un láser pulsado con una longitud de onda que oscila entre 650 y 950 nm (el más común es de $904 \mathrm{~nm}$ ).

- El láser de He-Ne emite dentro del espectro visible, concretamente del rojo a $632,8 \mathrm{~nm}$.

En la actualidad también se está comercializando un láser con longitud de onda de $635 \mathrm{~nm}$ (color rojo) bajo el nombre de Diagnodent $\AA$ (Kavo, Biverach, Alemania) (figs. 4 y 5). Más adelante se comentarán las aplicaciones de todos ellos.

Por otro lado, también se puede usar el láser de diodos (láser de alta potencia) con la pieza de mano para la aplicación del blanqueamiento dental, donde la potencia no excede de 1W (figs. 6 y 7). Otra aplicación puede estar dirigida, por ejemplo, a minimizar la inflamación postoperatoria tras una intervención quirúrgica. Este hecho puede definirse como característico de un láser de baja potencia.

\section{Aplicaciones clínicas}

\section{Terapéutica Dental}

Por la posibilidad de obtener el efecto analgésico y antiinflamatorio, el láser de baja potencia puede estar indicado para disminuir el dolor que aparece tras los traumatismos dentarios, en la patología inflamatoria peria-

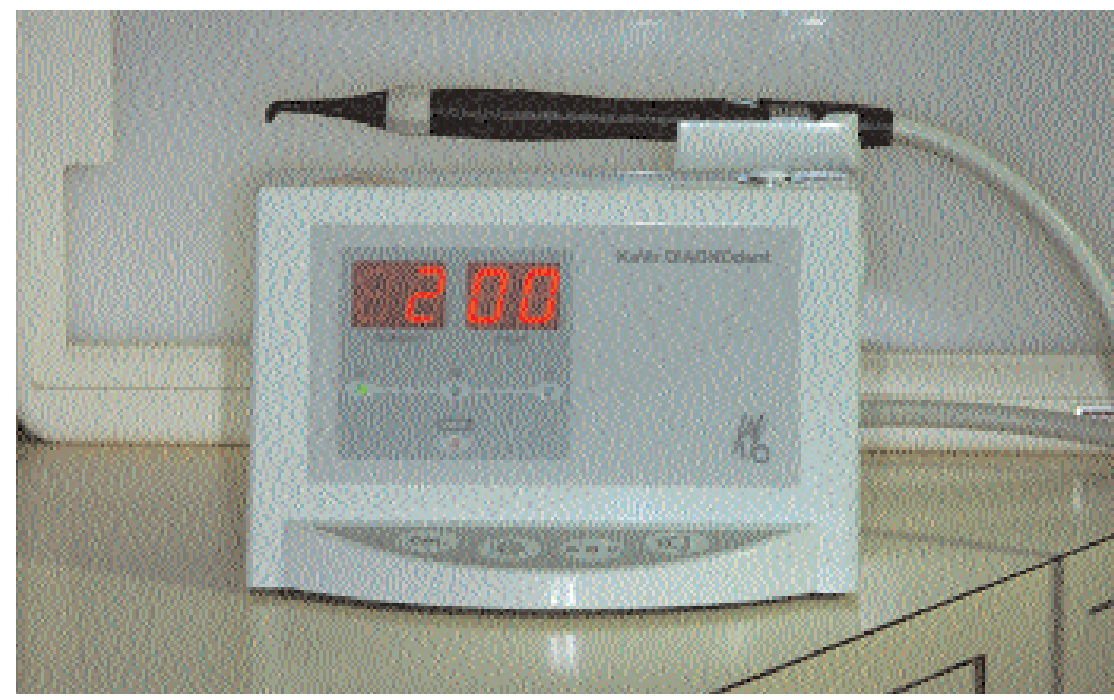

Figura 4: DIAGNOdent ${ }^{\circledR}$.

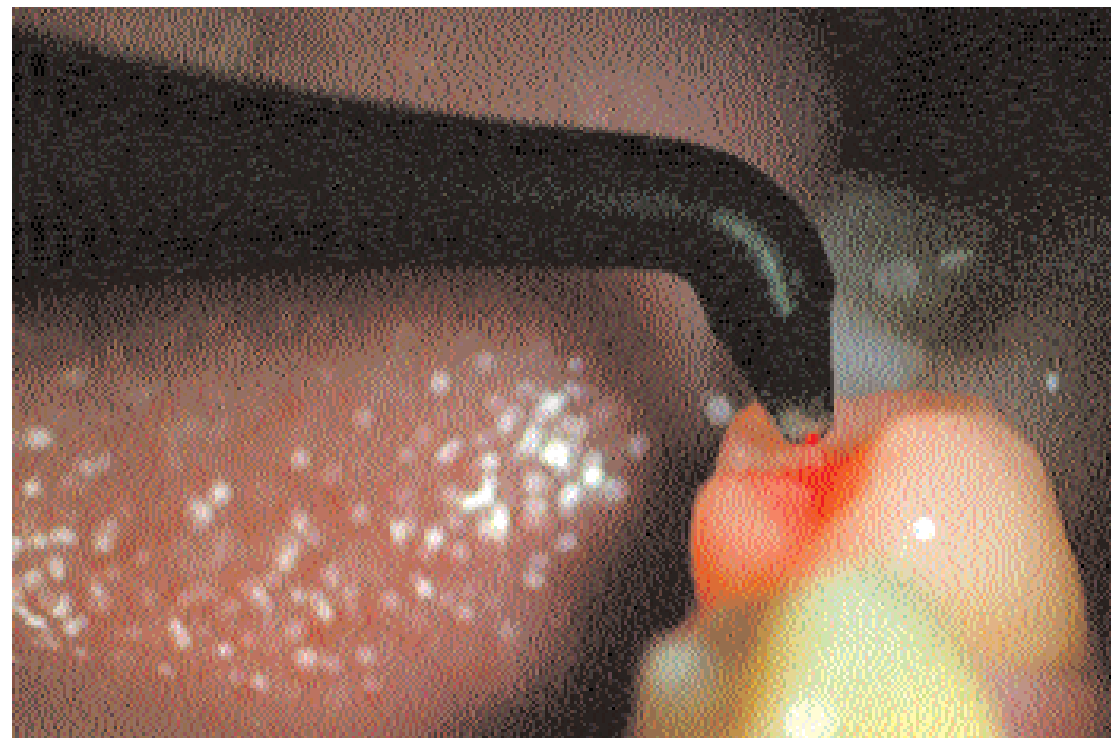

Figura 5: Aplicación del DIAGNOdent ${ }^{\circledR}$ para descubrir caries tempranas.

pical y en el postoperatorio de pacientes intervenidos de cirugía periapical, con resultados positivos en todos los $\operatorname{casos}^{5}$. Se debe utilizar como complemento al tratamiento farmacológico clásico y no como un tratamiento alternativo.

En el tratamiento de la hiperestesia dentinaria se han descrito resultados satisfactorios, comparables a los obtenidos con el barniz de flúor, no encontrándose diferencias estadísticamente significativas entre la administración de diferentes niveles de energía ${ }^{6,7}$. Se obtuvieron mejores resultados en los casos de hiperestesia más acusada, si bien autores como Wilder-Smith ${ }^{8 \star}$ no describen ventajas en estos casos. 


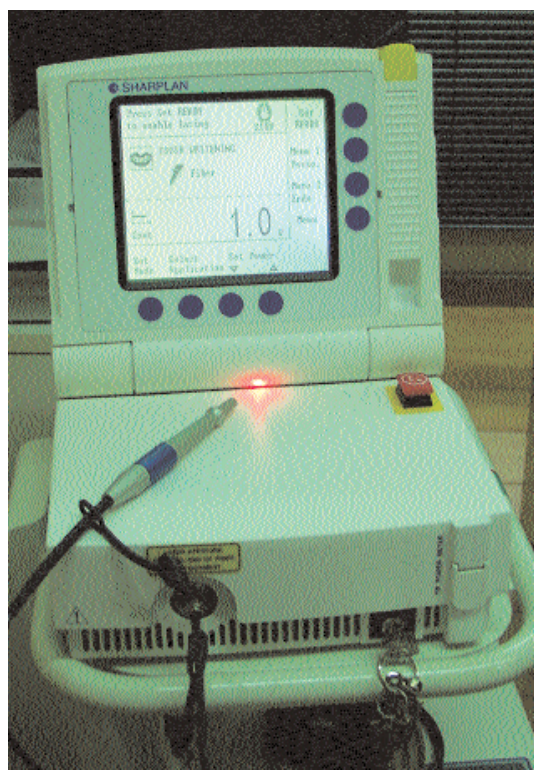

Figura 6: Láser de diodo con pieza de mano para el blanqueamiento dental.

Otros autores han efectuado estudios in vitro para valorar la aplicación del láser blando como método de desinfección de los conductos radiculares junto con hipoclorito sódico $(\mathrm{NaOCl})$ y peróxido de hidrógeno $\left(\mathrm{H}_{2} \mathrm{O}_{2}\right)$. Sus resultados son muy esperanzadores ${ }^{9}$.

Otra aplicación de los láseres de baja energía es la posibilidad de diagnosticar las caries tempranas y éste es el caso de un láser comercializado bajo el nombre de DIAGNOdent ${ }^{\circledR}$ (Kavo, Biverach, Alemania) (figs. 4 y 5). El mecanismo que utiliza se basa en la transmisión de la fluorescencia en los tejidos dentarios; se hace incidir la luz láser y el aparato evalúa la fluorescencia del esmalte y de la dentina. La pieza de mano que utiliza puede escanear superficies oclusales, zonas proximales, etc., con una gran versatilidad y sin dificultades. Autores como Takamori y cols $^{10}$ confirman que puede ser útil para el diagnóstico de caries pero encuentran falsos positivos al irradiar sobre la placa bacteriana, el cálculo, en zonas con restos alimentarios y sobre las obturaciones con composites. Por tanto tiene una elevada sensibilidad pero una baja especificidad $^{11}$.

\section{Periodoncia}

La mayoría de los estudios publica-

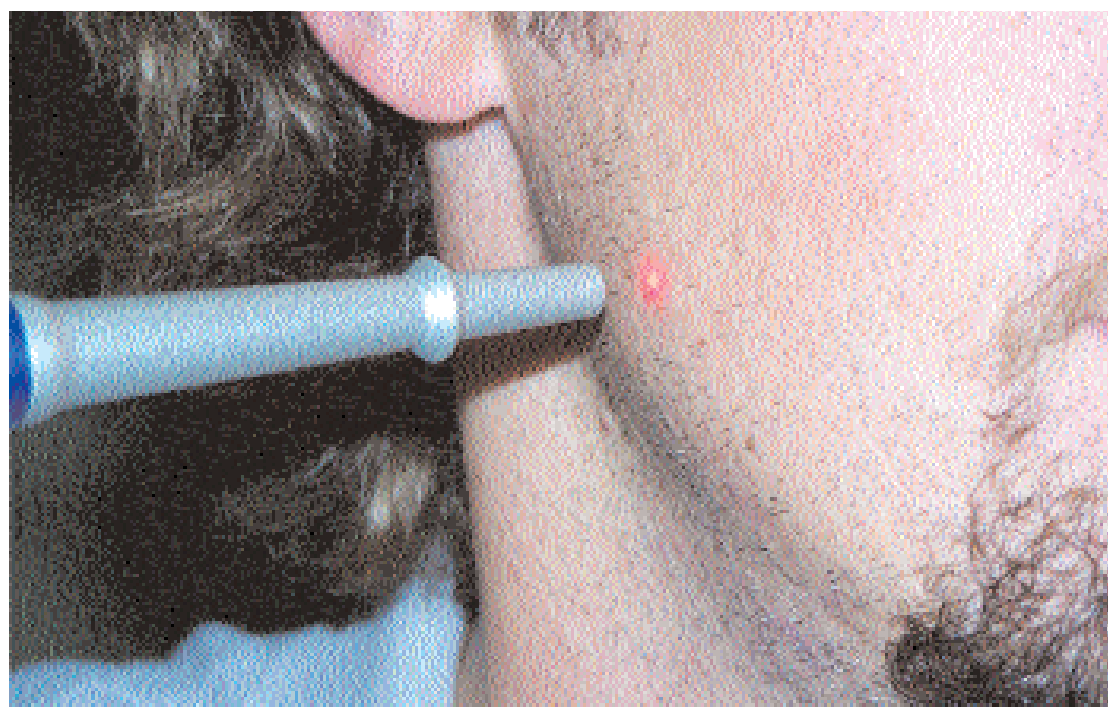

Figura 7: Aplicación del láser de diodo con la pieza de mano de baja potencia para minimizar la inflamación postoperatoria. dos acerca de las aplicaciones del láser en este campo, se centran en las ventajas de los láseres de alta potencia en cirugía periodontal ${ }^{12 *}$.

Sin embargo, existen otros estudios in vitro ${ }^{13} \mathrm{e}$ in vivo ${ }^{14}$ que han intentado demostrar la utilidad del láser blando en relación con la proliferación de los fibroblastos del ligamento periodontal humano. En éstos se concluye que existe mayor actividad proliferativa celular y que se necesitan más estudios que profundicen sobre estos hallazgos.

Otros autores han aplicado la tecnología del láser blando en el control del dolor postoperatorio después de efectuar curetajes periodontales ${ }^{1}$ observando disminución del dolor mediante escalas analógicas visuales. Sin embargo, Masse y cols $^{15}$ afirman que no existen diferencias significativas en el índice de curación y la reducción del dolor, si se compara con un tratamiento placebo. De este modo, los autores concluyen que el láser blando no es efectivo en el control del dolor tras los tratamientos quirúrgicos periodontales.

\section{Implantología bucofacial}

Numerosos autores han estudiado los efectos de la irradiación con láser de baja energía tras la colocación de implantes osteointegrados, aprovechando su carácter bioestimulante, para disminuir la inflamación, controlar el dolor postoperatorio y para favorecer la rápida cicatrización.

En experimentación animal se han estudiado otras variables, como la cantidad y la calidad ósea alrededor del implante, entre otros extremos. De este modo, en el estudio de Dortbudak y cols $^{16}$, tras irradiar con el láser de baja potencia las zonas periimplantarias de la cresta ilíaca en monos, inmediata- 
mente después de la colocación de implantes, se observó un mayor número de osteocitos viables por unidad de área respecto a las zonas no irradiadas o zonas control. Estos resultados se ven reforzados por el estudio de Guzzardella y cols $^{17}$ en el que se irradiaron las zonas periimplantarias en el fémur de conejos, observando mayor viabilidad ósea en la interfase entre el hueso y los implantes. Sin embargo, es necesario la realización de otros estudios clínicos que apoyen estas conclusiones.

De igual forma Kreisler y cols ${ }^{18^{*}}$ han publicado un estudio con este tipo de láser a fin de determinar su actividad antimicrobiana sobre las superficies de los implantes. Para ello hicieron cultivos de S. sanguinis sobre discos de titanio con diferentes tratamientos de superficie y midieron el crecimiento bacteriano tras irradiar con el láser de Ga,Al,As. Los resultados que se obtuvieron en este estudio determinaron una disminución del crecimiento bacteriano más o menos acusada en función del tipo de superficie. No obstante, la descontaminación es menor que la obtenida tras la inmersión de los implantes en clorhexidina durante un minuto. Por este motivo, estos autores concluyeron que la desinfección de las superficies im-plantarias mediante el uso de los láseres de baja energía es inferior a la obtenida mediante la descontaminación convencional in vitro

\section{Prótesis}

El uso del láser de baja potencia está indicado tras la cirugía preprotésica (apartado de Cirugía Bucal) y ante la presencia de úlceras por decúbito de las prótesis (apartado de Medicina Bucal) aprovechando sus características bioes- timulantes y favoreciendo así un mejor postoperatorio y/o curación.

\section{Ortodoncia}

Algunos autores han aprovechado las propiedades analgésicas del láser de baja potencia para disminuir el dolor tras los ajustes ortodóncicos. Este es el caso del estudio publicado por Lim y cols $^{19}$ donde los resultados demuestran un menor nivel de dolor en la escala analógica visual comparando con el grupo control pero sin encontrar diferencias estadísticamente significativas. Los autores concluyen que el uso del láser blando es un buen tratamiento coadyuvante de la terapia farmacológica clásica, pero no es suficiente como terapia alternativa.

Autores como Paulin y Vukicevic ${ }^{20^{*}}$ han determinado, gracias al uso del láser de He-Ne y un estudio holográfico, las reacciones que se producen en el esqueleto bucomaxilofacial tras el uso de un tornillo de expansión ortodóncico convencional. Con este sistema demostraron el desplazamiento de los huesos cigomáticos, los procesos pterigoideos o los huesos nasales.

\section{Cirugía Bucal}

Como se ha comentado anteriormente, los láseres de baja potencia carecen de efecto térmico pero sí que tienen un importante efecto celular bioestimulante. De este modo, su aplicación no estará indicada en el campo quirúrgico. Sin embargo, los láseres de baja potencia pueden ser aplicados, gracias a sus características, para acelerar la regeneración tisular y favorecer la cicatrización de las heridas consiguiendo una disminución de la inflamación y el dolor ${ }^{1}$. También han sido utilizados para potenciar la regeneración nerviosa tras la lesión de los nervios dentario inferior y lingual al demostrarse la recuperación de la sensibilidad de la zona afectada (fig. 2). Los resultados obtenidos en los diversos estudios publicados son muy variados y, quizás por este motivo, los autores no se atreven a generalizar los parámetros ideales para su utilización. No obstante, hay autores $^{21}$ que niegan todos estos resultados, muy probablemente por la dificultad de unificar criterios a la hora de medir parámetros subjetivos tan difíciles de cuantificar.

En este apartado es interesante destacar también la interacción que la palabra «láser» puede ejercer sobre la mente de los pacientes. De este modo puede actuar como placebo por las reminiscencias que en sí conlleva, y favorecer un mejor cumplimiento de las instrucciones postoperatorias ${ }^{22}$.

\section{Medicina Bucal}

El uso del láser de baja potencia también ha sido relacionado con el control de diversas lesiones de la mucosa bucal como las queilitis, quemaduras, la prevención de cicatrices hipertróficas y queloides, la mucositis por radio o quimioterapia, las aftas y el herpes, entre otras ${ }^{23}$.

Autores como Wong y cols $^{24}$ han observado con el uso del láser blando la reducción en la incidencia y severidad de la mucositis producida por la quimioterapia. Sin embargo, afirman desconocer el mecanismo que lo provoca y por este motivo, los parámetros ideales para su mayor optimización.

Por otro lado, Almeida-Lopes ${ }^{25^{*+}}$ afirma que el uso del láser de baja potencia disminuye el tiempo de curación del herpes simple labial y del herpes zóster. También concluye que, actuando precozmente (periodo prodrómico), puede 
llegar a disminuir la incidencia y la virulencia de estas infecciones víricas tan comunes. Siguiendo con esta misma publicación, Almeida-Lopes también nos ilustra sobre la posibilidad del uso del láser de baja potencia en el drenaje estimulado de adenopatías o de otras lesiones linfáticas. En este trabajo, básicamente clínico, la autora comenta las técnicas de drenaje linfático por cadenas y/o regiones y los puntos de aplicación del láser de una forma muy didáctica ${ }^{25^{*+}}$.

Patología disfuncional de la articulación temporomandibular (ATM) y dolor bucofacial

Como ha sido comentado ampliamente, el láser de baja potencia es utilizado para disminuir el dolor y la inflamación. En este sentido y dentro de la patología disfuncional de la ATM, autores como Jiméne $z^{26}$ han utilizado esta tecnología para contrarrestar la sintomatología álgica y disminuir el trismo con el que pueden cursar estas entidades nosológicas, y sus resultados son muy prometedores.

De igual forma, Kulekcioglu y cols $^{27}$ confirman estos resultados y concluyen que el uso del láser blando en la disfunción craneomandibular es un buen método alternativo ya que reduce el dolor y aumenta la apertura bucal y las lateralidades mandibulares.

En esta misma línea Pinheiro y cols $^{28,29}$ concluyen que el uso del láser de baja potencia es un método efectivo y beneficioso en el tratamiento de gran cantidad de alteraciones de la región maxilofacial como el dolor articular, la neuralgia del trigémino y el dolor muscular entre otros.

\section{Efectos secundarios}

Dentro de los efectos adversos, poco frecuentes, que puede provocar el uso del láser de baja potencia podemos encontrar, en primer lugar, el aumento del dolor que generalmente cede en la segunda sesión ${ }^{1}$.

Del mismo modo, otra complicación a tener en cuenta por las posibles consecuencias es la aparición de somnolencia y vértigo durante la aplicación en la patología disfuncional de la articulación temporomandibular ${ }^{26}$.

Finalmente es necesario remarcar que existen muy pocos estudios que hagan referencia a los efectos adversos que puede producir la aplicación del láser de baja potencia.

\section{Contraindica- ciones}

Mier $^{1}$ y Basford ${ }^{30}$ enumeran una lista de contraindicaciones absolutas y relativas para el uso de la tecnología láser en Odontología que se detalla a continuación:

1. Absolutas:

- Irradiación directa e indirecta sobre el globo ocular.

- Irradiación de la glándula tiroides.

- Pacientes con neoplasias.

- Pacientes epilépticos.

- Pacientes con mastopatía fibroquística.

- Irradiación prolongada en niños en edad de crecimiento.

- Pacientes que llevan marcapasos.

- Pacientes con infarto de miocardio reciente.

2. Relativas:

- Distiroidismo.

- Embarazo.

- Infecciones bacterianas sin previa cobertura antibiótica.

- Combinación con fármacos que producen fotosensibilidad.

- Pieles fotosensibles.

- Dolor de origen orgánico o visceral.

Algunas de las contraindicaciones citadas anteriormente están basadas en relación al efecto bioestimulante que posee este láser y a las hipotéticas consecuencias que habría que esperar sobre las células germinativas tanto benignas como malignas.

\section{Bibliografía recomendada}

Para profundizar en la lectura de este tema, el/los autor/es considera/an interesantes los artículos que aparecen señalados del siguiente modo: *de interés ${ }^{*}$ de especial interés.

1. Mier M. Laserterapia y sus aplicaciones en Odontología. Práctica Odontológica 1989; 10:9-16.

2. Midda M, Renton-Harper P. Lasers in dentistry. Br Dent J 1991;70:343-6.
3. Sliney DH, Trokel SL. Medical lasers and their safe use. New York: Springer-Verlag,1993. 4.** Trullols Casas C, España Tost A, Berini Aytés L, Gay Escoda C. Aplicaciones del láser blan- do en Odontología. Anal Odontoestomatol 1997;2:45-51.

Artículo muy completo y de lectura obligatoria donde se ilustran las principales características, 
usos y contraindicaciones del láser de baja potencia tras una exhaustiva revisión.

5. Kreisler MB, Haj HA, Noroozi N, Willershausen $B$. Efficacy of low level laser therapy in reducing postoperative pain after endodontic surgery: A randomized double blind clinical study. Int J Oral Maxillofac Surg 2004;33:38-41.

6. Marsilio AL, Rodrigues JR, Borges AB. Effect of the clinical application of the GaAlAs laser in the treatment of dentine hypersensitivity. J Clin Laser Med Surg 2003;21:291-6.

7. Corona SAM, Nascimento TNd, Catirse ABE, Lizarelli RF, Dinelli W, Palma-DIBB RG. Clinical evaluation of low-level laser therapy and fluoride varnish for treating cervical dentinal hypersensitivity. J Oral Rehab 2003;30:1183-9.

8.* Wilder-Smith P. The soft laser: Therapeutic tool or popular placebo? Oral Surg Oral Med Oral Pathol 1988;66:654-8.

Artículo interesante por su enfoque crítico sobre las aplicaciones del láser de baja potencia en Odontología.

9. Kreisler M, Kohen W, Beck M y cols. Efficacy of $\mathrm{NaOCl} / \mathrm{H}_{2} \mathrm{O}_{2}$ irrigation and $\mathrm{GaAlAs}$ laser in decontamination root canals in vitro. Laser Surg Med 2003;32:189-96.

10. Takamori K, Hokari N, Okumura Y, Watanabe $\mathrm{S}$. Detection of occlusal caries under sealants by use of a laser fluorescence system. J Clin Laser Med Surg 2001;19:267-71.

11. Morgan M. Dental laser identifies early stages of caries. Dent Today 2000;19:30-3.

12.* Hoexter DL. Latest advances in laser systems and periodontal surgery. Dent Clin North Am 2001;45:207-12.

Artículo de lectura necesaria por la exhaustiva y contemporánea revisión llevada a cabo sobre varios tipos de láseres, especialmente en su aplicación en cirugía periodontal.

13. Kreisler M, Christoffers AB, Willershausen B, d'Hoedt B. Effect of low-level GaAlAs laser irradiation on the proliferation rate of human periodontal ligament fibroblasts: an in vitro study. J Clin Periodontol 2003;30:3538.

14. Almeida-Lopes L, Rigau J, Zangaro RA, Guidugli-Neto J, Jaeger MM. Comparison of the low level laser therapy effects on cultured human gingival fibroblasts proliferation using different irradiance and same fluence. Lasers Surg Med 2001;29:179-84.

15. Masse JF, Landry RG, Rochette C, Dufour L, Morency R, D'Aoust P. Effectiveness of soft laser treatment in periodontal surgery. Int Dent J 1993;43:121-7.

16. Dortbudak O, Haas R, Mailath-Pokorny G. Effect of low-power laser irradiation on bony implant sites. Clin Oral Implants Res 2002;13:288-92.

17. Guzzardella GA, Torricelli P, Nicoli-Aldini N, Giardino R. Osseointegration of endosseous ceramic implants after postoperative low-power laser stimulation: An in vivo comparative study. Clin Oral Implants Res 2003;14:226-32.

18.* Kreisler M, Kohen W, Marinello C y cols. Antimicrobial efficacy of semiconductor laser irradiation on implant surfaces. Int J Oral Maxillofac Implants 2003;18:706-11.

Los autores demuestran el efecto antimicrobiano que tiene el láser de baja potencia sobre los implantes dependiendo del tipo de superfície aunque, lamentablemente, sin ventajas clínicas.

19. Lim HM, Lew KK, Tay DK. A clinical investigation of the efficacy of low level laser therapy in reducing orthodontic postadjustment pain. Am J Orthod Dentofacial Orthop 1995; 108:614-22.

20.* Pavlin D, Vukicevic D. Mechanical reactions of facial skeleton to maxillary expansion determined by laser holography. Am J Orthod 1984;85:498-507.

Artículo de elevado interés para los ortodoncistas. Sus autores descubren las reacciones que se producen en el esqueleto maxilofacial tras provocar una expansión del paladar con un tornillo de expansión convencional mediante el láser de He-Ne y un estudio holográfico.
21. Carrillo JS, Calatayud J, Manso FJ, Barberia E, Martinez JM, Donado M. A randomized double-blind clinical trial on the effectiveness of Helium-Neon laser in the prevention of pain, swelling and trismus after removal of impacted third molars. Int Dent J 1990;40:31-6.

22. Wigdor HA. Evaluation of the perception of patients of lasers and how lasers can make a dental visit easier. Lasers Surg Med 1995; Supple 7:15.

23. Velez M, Rigau J, Planas M. Láser de baja densidad de potencia en Dermatología. En: Cisneros Vela JL, Camacho Martínez F, (eds). Láser y fuentes de luz pulsada intensa en dermatología y dermocosmética. Madrid: Aula Medica; 2000. p. 303-11.

24. Wong SF, Wilder-Smith P. Pilot study of laser effects on oral mucositis in patients receiving Chemotherapy. Cancer J 2002;8:247-54.

25.** Almeida-Lopes L. Laserterapia na Odontologia. Biodonto 2004;1:9-87.

La autora realiza un exhaustivo monográfico sobre el láser de baja potencia donde se incluyen las aplicaciones, las pautas y la posología de su uso entre otros apartados.

26. Jiménez López V. El láser en el tratamiento de las disfunciones de la ATM. Rev Actual Odontoestomatol Española 1986;46:35-40.

27. Kulekcioglu S, Sivrioglu K, Ozcan O, Parlak M. Effectiveness of low-level laser therapy in temporomandibular disorder. Scand J Rheumatol 2003;32:114-8.

28. Pinheiro AL, Cavalcanti ET, Pinheiro TI, Alves MJ, Manzi CT. Low-level laser therapy in the management of disorders of the maxillofacial region. J Clin Laser Med Surg 1997;15:181-3.

29. Pinheiro AL, Cavalcanti ET, Pinheiro TI, Alves MJ, Miranda ER, De Quevedo AS y cols. Lowlevel laser therapy is an important tool to treat disorders of the maxillofacial region. J Clin Laser Med Surg 1998;16:223-6.

30. Basford JR. Low intensity laser therapy. Still not an established clinical tool. Lasers Surg Med 1995;16:331-42. 